\section{Rate-Type Concrete Creep LaW WITH REDUCED TIME}

By Zdeněk P. Bažant, ${ }^{1}$ F. ASCE and Jenn-Chuan Chern ${ }^{2}$

Abstract: For creep analysis of large structural systems, the linear aging integral-type creep law needs to be converted to a rate-type form which consists of a system of first-order linear differential equations with age-dependent coefficients. The system may be visualized by the Kelvin chain model with agedependent elastic moduli and viscosities. In the existing formulation, the independent variable is actual time. It is shown that, by using as the independent variable a certain reduced time which increases with time at a gradually declining rate, one reduces the number of differential equations needed to describe creep within the given time range, thereby making numerical structural analysis more efficient. An algorithm for identifying the material parameters from given creep data, based on minimization of a sum of squared deviations, is also presented. Thermodynamic restrictions on the material coefficients are analyzed. Finally, the capability of closely approximating available creep data is demonstrated.

\section{INTRODUCTION}

For numerical creep analysis of large structural systems, particularly finite element analysis, the rate-type form of a linear aging creep law is far more efficient than the integral-type form based directly on the principle of superposition. Instead of storing during step-by-step computation the values of stresses or strains in all previous steps, it suffices to store only the current values of several internal variables. The number of arithmetic operations also gets significantly reduced $(3,5,6,10,11,19)$.

The existing rate-type formulation of the aging creep law of concrete consists of a system of first or second-order linear differential equations with the actual time as an independent variable. This paper examines the question whether the rate-type formulation can be made more efficient by means of introducing as the independent variable a certain monotonically increasing function of time, called the reduced time.

Linear Aging Creep Law Based on Principle of Superposition.--For stresses less than about one-half of the strength, the creep law of concrete may be approximately considered as linear, i.e., obeying the principle of superposition. The linearity assumption greatly simplifies structural analysis, and is generally used in practice. According to the principle of superposition, the uniaxial creep law of concrete may be written in the form

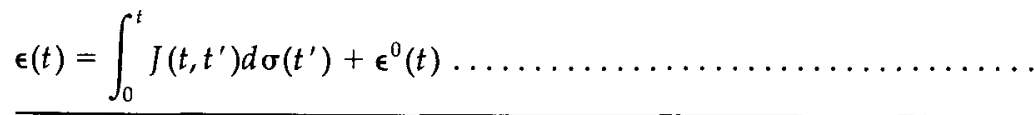

${ }^{1}$ Prof. of Civ. Engrg. and Dir,, Center for Concrete and Geomaterials, Technological Inst., Northwestern Univ., Evanston, IIl. 60201.

${ }^{2}$ Grad. Research Asst., Northwestern Univ., Evanston, Ill. 60201.

Note.-Discussion open until August 1,1984. To extend the closing date one month, a written request must be filed with the ASCE Manager of Technical and Professional Publications. The manuscript for this paper was submitted for review and possible publication on January 7, 1983. This paper is part of the Journal of Engineering Mechanics, Vol. 110, No. 3, March, 1984. OASCE, ISSN 07339399/84/0003-0329/\$01.00. Paper No. 18621. in which $\sigma, \epsilon=$ uniaxial stress and strain; $\epsilon^{0}=$ prescribed stress-independent strain representing shrinkage and thermal dilatation; and $J\left(t, t^{\prime}\right)$ $=$ compliance function (also called creep function) $=$ strain at age $t$ caused by a unit uniaxial stress acting since age $t^{\prime}$.

Conversion of Creep Law to Rate-Type Form.-When strain $\epsilon(t)$ is unknown, Eq. 1 represents a Volterra integral equation. As is well known, this equation may be converted to a system of differential equations when the kernel $J\left(t, t^{\prime}\right)$ has the so-called degenerate form, which consists of a sum of products of functions of $t$ and functions of $t^{\prime}$. This may be written as $J\left(t, t^{\prime}\right)=\Sigma_{\mu}\left[1 / C_{\mu}\left(t^{\prime}\right)\right]-\Sigma_{\mu}\left[B_{\mu}(t) / B_{\mu}\left(t^{\prime}\right) C_{\mu}\left(t^{\prime}\right)\right]$ where $C_{\mu}$ and $B_{\mu}$ are functions of time, and $\mu=1,2, \ldots N$. It is convenient to set $B_{\mu}(t)$ $=\exp \left[-y_{\mu}(t)\right]$, in which $y_{\mu}(t)=$ functions of time representing what will be called the reduced times. Then, the general form of a degenerate kernel may be written as

$J\left(t, t^{\prime}\right)=\sum_{\mu=1}^{N} \frac{1}{C_{\mu}\left(t^{\prime}\right)}\left\{1-e^{\left[y_{\mu}\left(t^{\prime}\right)-y_{\mu}(t)\right]}\right\}$

This is a series of real exponentials, called Dirichlet series or Prony series $(12,16,17,21,23)$. Substituting Eq. 2 into Eq. 1, we obtain

$\epsilon(t)=\sum_{\mu=1}^{N} \epsilon_{\mu}(t)+\epsilon^{0}(t)$

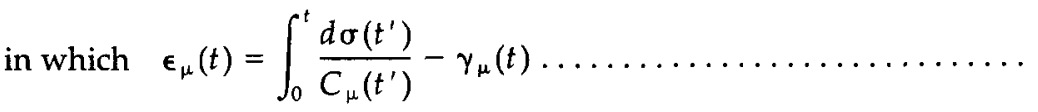

$\gamma_{\mu}(t)=e^{\left[-y_{\mu}(t)\right]} \int_{0}^{t} e^{\left[y_{\mu}\left(t^{\prime}\right)\right]} \frac{d \sigma\left(t^{\prime}\right)}{d y_{\mu}\left(t^{\prime}\right)} \frac{d y_{\mu}\left(t^{\prime}\right)}{C_{\mu}\left(t^{\prime}\right)}$

It may now be checked by substitution into Eqs. 3 and 1 that the following linear differential equations are always satisfied by the preceding expression for $\epsilon_{\mu}$ :

$\frac{d^{2} \epsilon_{\mu}}{d y_{\mu}^{2}}+\frac{d \epsilon_{\mu}}{d y_{\mu}}=\frac{1}{C_{\mu}(t)} \frac{d \sigma}{d y_{\mu}} \quad(\mu=1,2, \ldots N)$

Expressing the derivative $d \gamma_{\mu} / d y_{\mu}$ from Eq. 5, we may verify that functions $\gamma_{\mu}$ always satisfy the differential equation

$\frac{d \gamma_{\mu}}{d y_{\mu}}+\gamma_{\mu}=\frac{1}{C_{\mu}(t)} \frac{d \sigma}{d y_{\mu}} \quad(\mu=1,2, \ldots N)$.

Variables $\epsilon_{\mu}$ and $\gamma_{\mu}$ are related to $\epsilon$ by the differential equations

$\dot{\boldsymbol{\epsilon}}=\sum_{\mu} \dot{\boldsymbol{\epsilon}}_{\mu}+\dot{\boldsymbol{\epsilon}}^{0} \ldots$

$\dot{\boldsymbol{\epsilon}}_{\mu}=\frac{\dot{\sigma}}{C_{\mu}(t)}-\dot{\gamma}$

It can be further shown that integration of Eq. 6 yields Eq. 4 for $\epsilon_{\mu}$ and that integration of Eq. 7 yields Eq. 5 for $\gamma_{\mu}$. This confirms that the rate-type creep law given either by Eqs. 6 and 8, or by Eqs. 7 and 8, is 
equivalent to the original integral-type creep law in Eq. 1. Note that functions $y_{\mu}(t)$ are treated in Eqs. 6 or 7 as independent variables, analogous to time. That is why $y_{\mu}(t)$ are called the reduced times. It is, of course, necessary that $y_{\mu}(t)$ be monotonically increasing functions of actual time $t$

The derivatives in Eq. 6 may be converted to time derivatives by substituting $d \epsilon_{\mu} / d y_{\mu}=\dot{\epsilon}_{\mu} / \dot{y}_{\mu}, d^{2} \epsilon_{\mu} / d y_{\mu}^{2}=\left(\ddot{\epsilon}_{\mu} \dot{y}_{\mu}-\dot{\epsilon}_{\mu} \ddot{y}_{\mu}\right) / \dot{y}_{\mu}^{2}$. This yields

$\ddot{\boldsymbol{\epsilon}}_{\mu}+\left(\dot{y}_{\mu}-\frac{\ddot{y}_{\mu}}{\dot{y}_{\mu}}\right) \dot{\boldsymbol{\epsilon}}_{\mu}=\frac{\dot{y}_{\mu}}{C_{\mu}(t)} \dot{\boldsymbol{\sigma}}$

It is convenient to interpret the form of this equation by means of a rheologic model. Examine the Kelvin chain model (Fig. 1), in which $\epsilon_{\mu}$ denotes the strain of the Kelvin unit number $\mu$, while $E_{\mu}$ and $\eta_{\mu}$ represent the spring modulus and dashpot viscosity of this unit, respectively. As has been shown before (4), the stress in the spring cannot be expressed as $E_{\mu}(t) \epsilon_{\mu}$ if the material is aging, or else the second law of thermodynamics would be violated. Rather, the rate of the stress in the spring is $E_{\mu}(t) \dot{\epsilon}_{\mu}$. The stress in the dashpot is $\eta_{\mu}(t) \dot{\epsilon}_{\mu}$, and its rate is $\left[\eta_{\mu}(t) \dot{\epsilon}_{\mu}\right]$. Thus, the rate of the stress carried by the $\mu$ th Kelvin unit is $\left[\eta_{\mu}(t) \dot{\boldsymbol{\epsilon}}_{\mu}\right]+E_{\mu}(t) \dot{\boldsymbol{\epsilon}}_{\mu}$, which must equal $\dot{\boldsymbol{\sigma}}$ according to Fig. $1(a)$. This furnishes the well-known equation $(2,10)$

$\ddot{\epsilon}_{\mu}+\frac{E_{\mu}(t)+\dot{\eta}_{\mu}(t)}{\eta_{\mu}(t)} \dot{\epsilon}_{\mu}=\frac{\dot{\sigma}}{\eta_{\mu}(t)}$

This differential equation is of second-order, whereas that for a nonaging material is of first-order.

Equating the coefficients of Eqs. 9 and 10, we find (2)

$\eta_{\mu}(t)=\frac{C_{\mu}(t)}{\dot{y}_{\mu}(t)}$

$E_{\mu}(t)=C_{\mu}(t)-\frac{\dot{C}_{\mu}(t)}{\dot{y}_{\mu}(t)}$

The phenomenon of aging that is relevant here is the gradual hardening of concrete due to continuing hydration. Its effect is to slow down
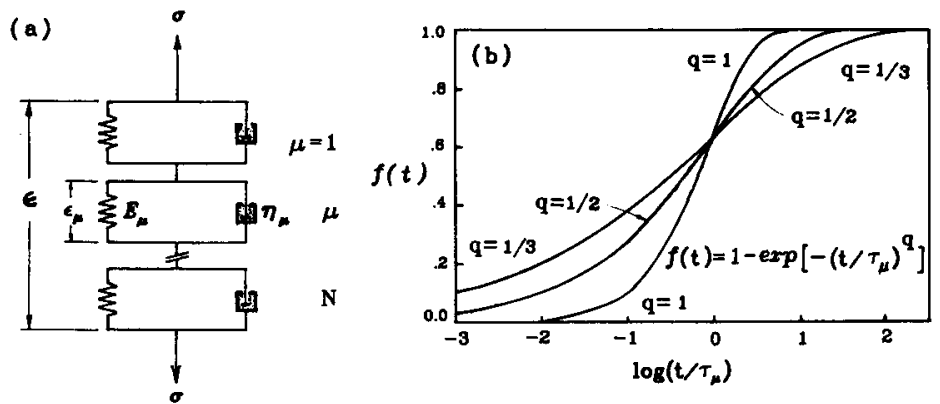

all rate phenomena, as if the time were running slower. This may be most simply described by setting

$y_{\mu}=\left(\frac{t}{\tau_{\mu}}\right)^{q}$ with $0<q \leq 1$

in which $q=$ constant; $\tau_{\mu}$ are constants called the retardation times. Eq. 11 then becomes

$\eta_{\mu}(t)=\tau_{\mu}^{a} C_{\mu}(t) \frac{t^{1-q}}{q}$

$E_{\mu}(t)=C_{\mu}(t)-\tau_{\mu}^{q} \dot{C}_{\mu}(t) \frac{t^{1-q}}{q}$

It may be concluded that a Kelvin chain model that is equivalent to the most general form of a degenerate kernel (Eq. 2) can be always be found.

Similarly, expressing the relaxation function as a Dirichlet series in reduced time, one can reduce the aging integral-type creep law based on the relaxation function to a system of first-order equations which are modeled by the Maxwell chain (6).

When $q=1$, the reduced times are proportional to the actual time and the present formulation becomes equivalent, as a special case, to the previously developed rate-type formulation based on Dirichlet series expansion $(3,10,11)$. Furthermore, if there is no aging, i.e., if $C_{\mu}$ are constant, the formulation becomes identical to that developed for nonaging materials by Zienkiewicz and Watson (24) and by Taylor, et al. (22).

Fitting of Given Creep Data.-A given compliance function $J\left(t, t^{\prime}\right)$ may be approximated by the Dirichlet series in Eq. 1 with any desired accuracy using a similar approach as that previously developed for the case $q=1(3,5,6,7,10,11)$. The retardation times $\tau_{\mu}$ cannot be calculated from test data but must be chosen in advance. Their suitable choice is a crucial aspect which was studied in depth $(3,5,6,8,10,11)$. The coefficients $C_{\mu}(t)$ may be obtained by minimizing the sum of squared deviations from given data.

For the special case $q=1$ used so far, i.e., $y_{\mu}=t / \tau_{\mu}$, a suitable choice of $\tau_{\mu}$ is known to be $\tau_{1}=10^{-20}, \tau_{\mu}=10^{\mu-2} \tau_{2}(\mu=2,3, \ldots N)$. The first retardation time is chosen to be negligibly small so that the first Kelvin unit would represent the instantaneous deformation. For programming this is more economical than using a separate spring to model the instantaneous deformation. Using $\tau_{\mu}=10^{\mu-2} \tau_{2}$, the retardation times are spaced uniformly in the logarithmic time scale, by decades. A sparser spacing produces unsmooth creep curves, and a denser spacing wastes computer time since no better representation of the creep curves is achieved.

In the present formulation, the rates are given with respect to the reduced times $y_{\mu}=\left(t / \tau_{\mu}\right)^{q}$ rather than the actual time $t$. When we change from the actual time $t$ to the reduced time $y_{\mu}$, the response curve of each Kelvin unit changes from $1-\exp \left(-t / \tau_{\mu}\right)$ to $1-\exp \left[-\left(t / \tau_{\mu}\right)^{q}\right]$, which is shown in Fig. 1(b). The breadth of the rise of the curve in Fig. $1(b)$ increases as the exponent $q$ gets smaller, and therefore the spacing of the retardation times in the logarithmic scale can be made larger. This 
is the main purpose of the present formulation. From Fig. 1(b) it is clear that the breadth of the rise is proportional to time to the power $q$. Therefore, the values that are uniformly spaced in the logarithmic time scale should be $\tau_{\mu}^{q}$ rather than $\tau_{\mu}$, and so $\tau_{\mu}^{q}=\tau_{2}^{q} a^{\mu-2}$, in which $a$ is a constant which should be empirically determined as the largest possible value to yield smooth creep curves. Thus

$\tau_{1}=10^{-20}$.

$\tau_{\mu}=a^{(\mu-2) / 9} \tau_{2} \quad(\mu=2,3, \ldots N)$

The values of retardation times $\tau_{\mu}$ must fully cover the complete range of times over which representation of creep is desired. In analogy to the previously established empirical rules for the case $q=1$, the following conditions must be met

$\tau_{2} \leq 0.6^{1 / q} \min \left(t-t^{\prime}\right)$

$\tau_{N} \geq 3^{1 / q} \max \left(t-t^{\prime}\right)$

$\tau_{2} \leq \min t^{\prime}$

in which $\min \left(t-t^{\prime}\right)$ and $\max \left(t-t^{\prime}\right)=$ the shortest and the longest creep durations for which representation of creep is desired, respectively, and $\min t^{\prime}=$ the smallest age at loading to be considered.

By analysis of numerous test data, it has been found that the maximum acceptable value of $a$ is 15 , but for smoothness of the response curves the following value is suitable:

$a=10$

As for the value of exponent $q$, the smaller it is the more efficient is the computation, however, for too small a value good fits of typical creep curves cannot be obtained. By analysis of test data; the minimum acceptable value has been found as

$q=\frac{2}{3}$

If the range of creep durations of interest spans from $t-t^{\prime}=0.02$ day to $t-t^{\prime}=10^{4}$ days, the required number of retardation times for $a=$ 10 and $q=1$ is $N=7$. Using $q=2 / 3$, the number of retardation times can be reduced to $N=5$, thus preserving equally good fits.

The deviations from test data are evaluated at chosen sampling points $\xi_{m}(m=1,2, \ldots)$ of creep duration $\xi=t-t^{\prime}$. These sampling points should be uniformly spaced in $\log t$-scale. The sampling points $t_{k}^{\prime}$ for the age at loading, $t^{\prime}$, should be uniformly spaced in $\log t^{\prime}$-scale. The values of $J\left(t, t^{\prime}\right)$ for the sampling times are, according to Eq. 2

$J\left(t_{k}^{\prime}+\xi_{m}, t_{k}^{\prime}\right)=\sum_{\mu=1}^{N} f_{\mu m}^{k} X_{\mu}^{k} \ldots$

in which $f_{\mu m}^{k}=1-e^{\left[y_{\mu}\left(t_{k}^{\prime}\right)-y_{\mu}\left(t_{k}^{\prime}+\xi_{m}\right)\right]}$

$X_{\mu}^{k}=\frac{1}{C_{\mu}\left(t_{k}^{\prime}\right)}$
The sum of squares to be minimized may then be written as

$\Phi=\sum_{k=1}^{K} \Phi_{k}+\Pi$

$\Phi_{k}=\sum_{m}\left(\sum_{\mu=1}^{N} f_{\mu m}^{k} X_{\mu}^{k}-J_{k m}\right)^{2}+\Pi$

$\Pi_{k}=w_{1} \sum_{\mu=2}^{N}\left(X_{\mu}^{k}-X_{\mu-1}^{k}\right)^{2}+w_{2} \sum_{\mu=3}^{N}\left(X_{\mu}^{k}-2 X_{\mu-1}^{k}+X_{\mu-2}^{k}\right)^{2}$

$\Pi=\sum_{\mu=1}^{N}\left[w_{1}^{\prime} \sum_{k=2}^{K}\left(X_{\mu}^{k}-X_{\mu}^{k-1}\right)^{2}+w_{2}^{\prime} \sum_{k=3}^{K}\left(X_{\mu}^{k}-2 X_{\mu}^{k-1}+X_{\mu}^{k-2}\right)^{2}\right]$

in which $J_{k m}=$ the measured or given creep compliance values at the sampling points; $\Pi, \Pi_{k}=$ penalty terms which penalize for unsmooth variation of $X_{\mu}^{k}$ with regard to $\mu$ and $k ; w_{1}, w_{2}=$ suitable (empirically) chosen weights which enforce smoothness of the variation of $X_{\mu}^{k}$ as a function of $\mu$; and $w_{1}^{\prime}, w_{2}^{\prime}$ are further chosen weights which enforce a smooth variation of $X_{\mu}^{k}$ as a function of $k$. The minimizing conditions are $\partial \Phi / \partial X_{v}^{l}=0(v=1, \ldots N ; l=1, \ldots K)$, which yield the equation system

$\sum_{k=1}^{K}\left[\sum_{m}\left(\sum_{\mu=1}^{N} f_{\mu m}^{k} X_{\mu}^{k}-J_{l m}\right) f_{\nu m}^{l}+w_{1} \sum_{\mu=2}^{N}\left(X_{\mu}^{l}-X_{\mu-1}^{l}\right)\left(\delta_{\nu}^{\mu}-\delta_{\nu}^{\mu-1}\right)\right.$

$\left.+w_{2} \sum_{\mu=3}^{N}\left(X_{\mu}^{l}-2 X_{\mu-1}^{l}+X_{\mu-2}^{l}\right)\left(\delta_{\nu}^{u}-2 \delta_{\nu}^{\mu-1}+\delta_{\nu}^{\mu-2}\right)\right]$

$+w_{1}^{\prime} \sum_{k=2}^{K}\left(X_{v}^{k}-X_{v}^{k-1}\right)\left(\delta_{l}^{k}-\delta_{l}^{k-1}\right)$

$+w_{2}^{\prime} \sum_{k=3}^{K}\left(X_{v}^{k}-2 X_{v}^{k-1}+X_{v}^{k-2}\right)\left(\delta_{l}^{k}-2 \delta_{l}^{k-1}+\delta_{l}^{k-2}\right)=0$

in which $\delta_{i}^{j}=1$ if $i=j$, and 0 if $i \neq j$. Eq. 20 is a system of linear equations from which $X_{\mu}^{k}$ may be solved.

If $w_{1}^{\prime}$ and $w_{2}^{\prime}$ are chosen 0 , the least-square fitting can be carried out separately for each creep curve at constant $t^{\prime}=t_{k}^{\prime}$. This approach has to be used when the spacing of sampling ages $t_{k}^{\prime}$ is uneven. The conditions of optimum fit then are $\partial \Phi_{k} / \partial X_{\mu}^{k}=0(\mu=1, \ldots N)$, which again yields a system of linear equations from which $X_{1}^{k}, \ldots X_{N}^{k}$ may be solved. In this case the variation of $X_{\mu}^{k}$ with $k$ can be quite unsmooth.

A smooth variation of $C_{\mu}\left(t^{\prime}\right)$ as a function of age $t^{\prime}$ may be achieved by introducing a linear combination of smooth monotonic functions, i.e. $1 / C_{\mu}\left(t^{\prime}\right)=\Sigma_{k} Y_{\mu}^{k} \phi_{k}\left(t^{\prime}\right)$, in which $\phi_{k}\left(t^{\prime}\right)$ are suitably chosen monotonically decaying functions, such as $\phi_{k}\left(t^{\prime}\right)=1, t^{\prime-1 / 8}, t^{\prime-2 / 8}, t^{\prime-3 / 8}$, or $\phi_{k}\left(t^{\prime}\right)$ $=\exp \left[-\lambda_{k} y_{k+c}\left(t^{\prime}\right)\right]$ in which $c=$ an integer value such that $\tau_{1+c}$ is of the same order order of magnitude as $t_{1}^{\prime}$. Forming the sum of squares 
$\Pi$ with the help of these expressions, and imposing the minimization conditions $\partial \Phi / \partial Y_{v}^{l}=0(v=1, \ldots N ; l=1, \ldots K)$, one obtains a system of linear equations for the unknown coefficients $Y_{\mu}^{k}$.

When the functions $1 / C_{\mu}\left(t^{\prime}\right)$ are characterized by their discrete values $X_{\mu}^{k}$, the intermediate values may be obtained by linear interpolation in the scale of $\log t^{\prime}$.

Fig. 2 shows the fits of various typical creep data available in the literature $(13,14,15,18,20)$. To eliminate the scatter, which is mostly of random nature, these data have been smoothed first with a double power log law (8). This new law for concrete creep at constant hygrothermal conditions represents an improvement over the double power law (6) in which the power curves of creep duration $t-t^{\prime}$ become straight lines in the $\log \left(t-t^{\prime}\right)$-scale at a certain critical value of strain (see the horizontal dash-dot lines in Fig. 2). The slope of the creep curves is continuous through the transition and the slope of all straight lines is the same. It has been shown elsewhere (8) that this law improves the description of creep at long times, and in particular gives more realistic final slopes of the creep curves. The smoothing by the double power law log is shown by the solid curves in Fig. 2. These curves have been used as the given compliance function $J\left(t, t^{\prime}\right)$ for the present analysis. The fits obtained are shown by the dashed curves, and it is seen that the deviations are negligible compared to the scatter of creep measurements. The values of $\tau_{2}, q, a$, and of the number of retardation times $N$ are given in the figures, and so are the parameters of the double power log law defined in Ref. 8.

The present formulation admits considering different values of $q$ for different retardation times $\tau_{\mu}$. The reduced time may then be redefined as $y_{\mu}=\left(t / \tau_{\mu}\right)^{q_{\mu}}$. For the values of $q_{\mu}$, an arithmetic progression, increasing or decreasing between the values 1.0 and $q$, has been tried in the fitting of test data. However, the fits did not get any better and most of them got worse.

Thermodynamic Restrictions and Other Questions.-It is generally accepted that not every imaginable formula for the compliance function is thermodynamically admissible. It is not known, however, how to check the thermodynamic restrictions directly on the function $J\left(t, t^{\prime}\right)$. It is known only how to check them for the rate-type creep law which corresponds to some rheologic spring-dashpot model (6). If the thermodynamic conditions are satisfied for the rheologic model, then they are satisfied for the function $J\left(t, t^{\prime}\right)$ which the rheologic model represents or approximates. If the thermodynamic conditions are violated by the rheologic model, they may, but need not, be violated by the function $J\left(t, t^{\prime}\right)$. To be sure of correctness, it is thus highly desirable, although not requisite, to satisfy the thermodynamic conditions by the rheologic model which approximates $J\left(t, t^{\prime}\right)$.

For a spring-dashpot model, the second law of thermodynamics requires that the spring moduli $E_{\mu}$ and dashpot viscosities $\eta_{\mu}$ be nonnegative. Furthermore, in the case of aging, the second law of thermodynamics requires (6) that $\dot{s}=E_{\mu}(t) \dot{e}$ rather than $s=E_{\mu}(t) e$, where $s$ and $e$ are the stress and strain in the spring, respectively. This condition is satisfied by the expressions in Eq. 11. Furthermore, thermodynamics also requires that, for an aging material, $\dot{E}_{\mu}$ be a nondecreasing function of
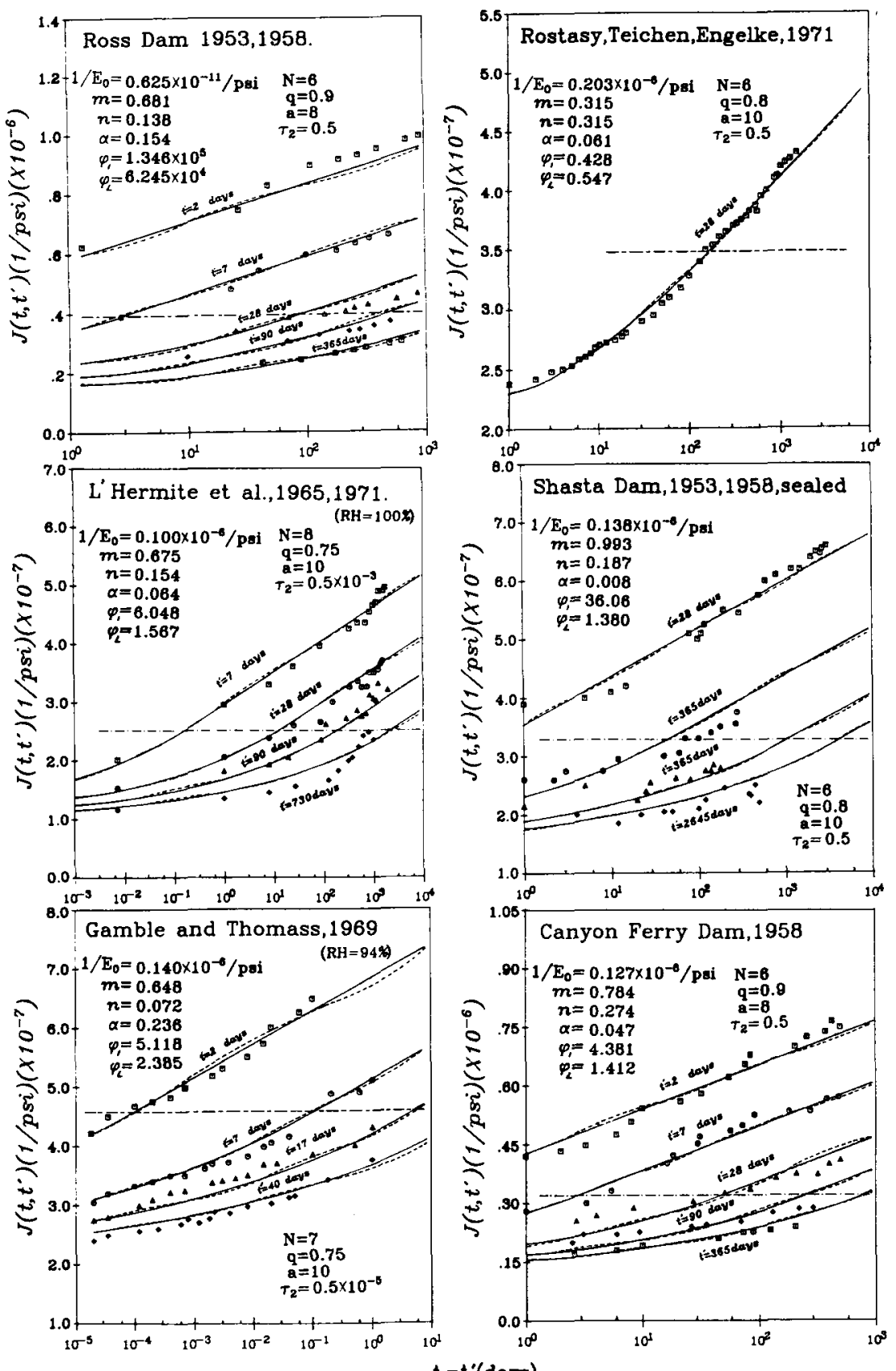

$8.0 \frac{10^{1}}{10^{2}} \frac{10^{3}}{\text { Shasta Dam, 1953,1958, sealed }}$
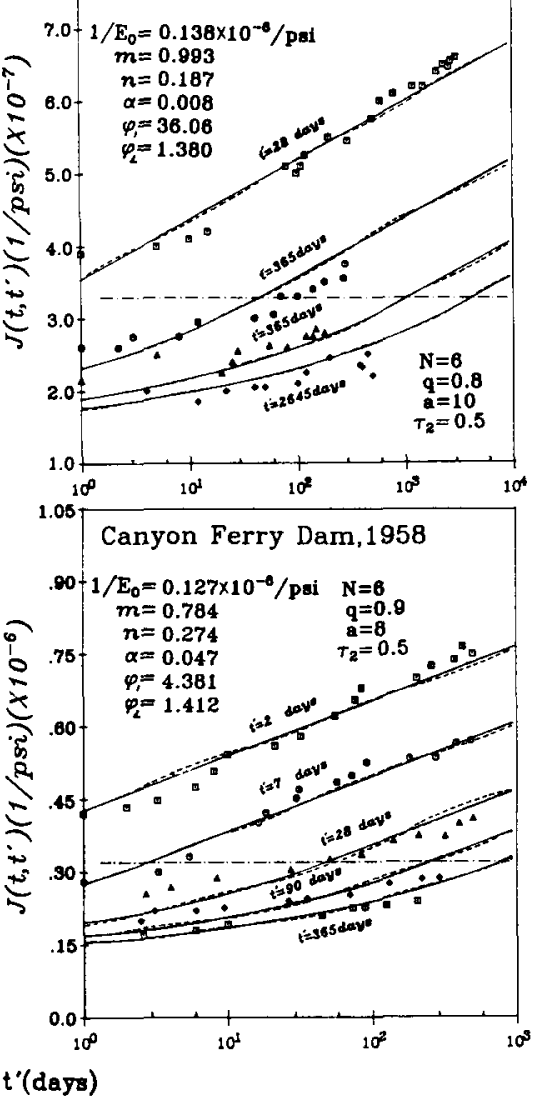

FIG. 2.-Smoothing of Test Data $(\mathbf{1 3 - 1 5 , 1 8 , 2 0 )}$-Solid Lines, and Fit with Present Theory-Dashed Lines 
time (6). Therefore, we should check for the present model whether the conditions $\eta_{\mu}>0, E_{\mu} \geq 0$ and $\dot{E}_{\mu} \geq 0$ are satisfied.

For the previous formulation with $q=1$, it has been found that, for the numerical values obtained by fitting typical creep data for the double power law, some of the foregoing conditions are always violated during some limited time intervals, even though they are satisfied most of the time. All the thermodynamic conditions could be satisfied with $q=1$ only for aging creep whose duration is not too long (4).

For the present formulation with $q<1$, it is found that violations of the foregoing thermodynamic conditions again cannot be avoided for aging creep of long durations. However, the occurrence of negative $E_{\mu}(t)$ or $E_{\mu}(t)$ is more limited than for $q=1$ and is restricted only to very short time intervals, always such time intervals for which the respective value of $E_{\mu}$ has little effect (e.g., the time intervals when the corresponding Kelvin unit has almost totally relaxed its stress). It may be also remarked that the occurrence of negative $E_{\mu}$ or negative $\dot{E}_{\mu}$ is less frequent for the double power log law than it is for the double power law. This might be due to the fact that the double power law exhibits the so-called divergence of creep curves, which can be avoided for the double power log law. Examples of the values of the spring moduli and dashpot viscosities, and their time dependence, are shown in Fig. 3. The only vi-
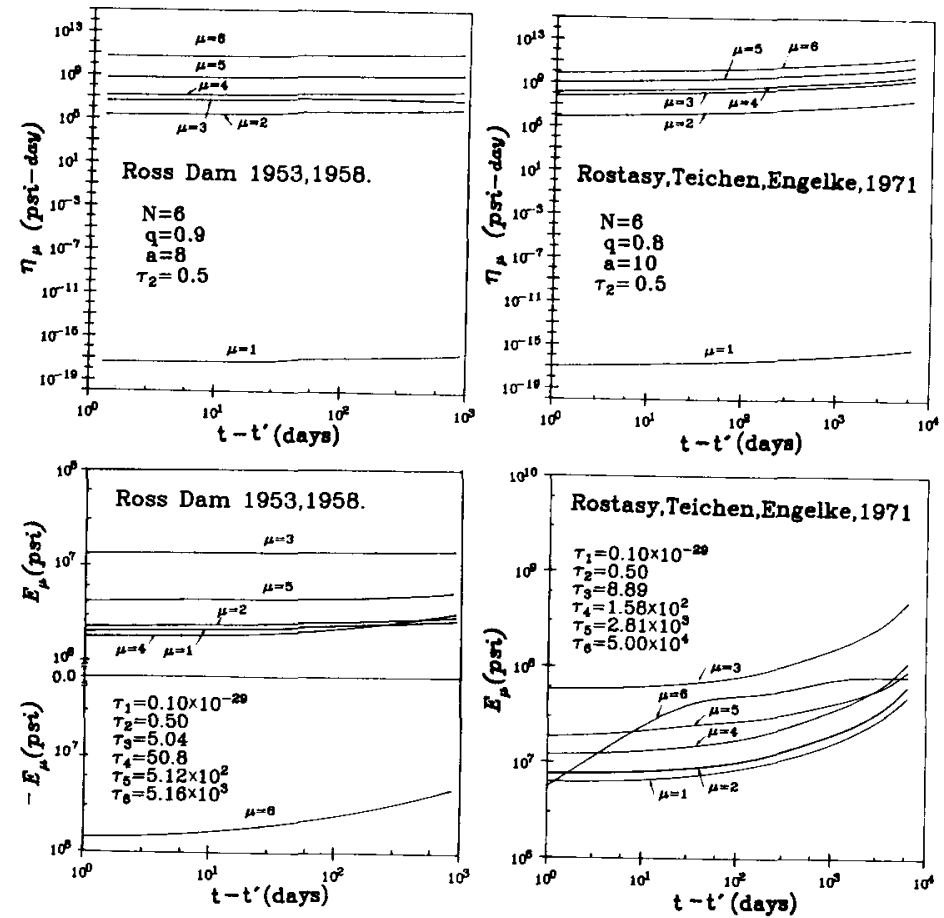

FIG. 3. -Time Variation of Viscosities and Elastic Moduli of Kelvin Chain Approximating Various Creep Data olation is in the negative values of $E_{\mu}$ at $\mu=6$, for the case of Ross Dam.

In view of the incomplete success of a persistent effort to assure fulfillment of all thermodynamic conditions, it now seems that the problem may be due to the fact that we are trying to represent with a linear creep law a phenomenon that is actually nonlinear. It is known that significant deviations from the principle of superposition occur even within the service stress range.

To be able to use the present formulation, an appropriate step-by-step numerical integration algorithm analogous to the well-known exponential algorithm for the case $q=1$ needs to be set up. This is done in Ref. 3.

\section{Conclusions}

1. Introduction of a reduced time whose rate of increase declines with age makes the creep law more efficient for use in structural analysis. Fewer retardation times are needed for the description of given creep data within the same time range.

2 . The retardation times are best spaced uniformly in the logarithm of the reduced time.

3. Least-square optimization can be used to identify the material parameters from given creep data.

4. Similarly to the previous linear creep models, the numerical values of elastic moduli obtained by fitting given long-time creep data violate occasionally the thermodynamic restrictions, even though the form of the rate-type creep law is correct from the thermodynamic viewpoint. These violations seem to be practically insignificant, and are fewer than for the existing rate-type creep law without reduced time.

5. Close fits of typical creep data from the literature are achieved.

\section{ACKNOWLeDgment}

Partial financial support under National Science Foundation Grant No. CEE-830-3148 to Northwestern University is gratefully acknowledged. Mary Hill is thanked for her meticulous typing.

\section{Appendix 1.-References}

1. Anderson, C. A. "Numerical Creep Analysis of Structures," Los Alamos Scientific Laboratory Report LA-UR-80-2585, Los Alamos, N.M., 1980; also “Creep entific Laboratory Report LA-UR-80-25," Z. P. Bažant, and F. H. Wittmann and Shrinkage in Concrete Structures, Z. P. Bažant, and F. H. W

eds., John Wiley \& Sons, Inc., New York, N.Y., 1982, pp. 259-304. Rheological Models," Acta Technica ČSAV, Prague 11, 1966, pp. 82-109.

3. Bažant, Z. P., "Theory of Creep and Shrinkage in Concrete Structures: A Précis of Recent Developments," Mechanics Today, Vol. 2, Pergamon Press, Inc., New York, N.Y., 1975, pp. 1-93.

4. Bažant, Z. P. "Viscoelasticity of Porous Solidifying Material-Concrete," Journal of the Engineering Mechanics Division, ASCE, Vol. 102, 1977, pp. 10491067. 
5. Bažant, Z. P., "Input of Creep and Shrinkage Characteristics for a Structural Analysis Program," Materials and Structures, RILEM, Paris, Vol. 15, No. 88 1982, p. 283; with Errata 1983, 1984.

6. Bažant, Z. P., "Mathematical Models for Creep and Shrinkage of Concrete," Chapter 7, Creep and Shrinkage in Concrete Structures, Z. P. Bažant, and F. H. Wittmann, eds., John Wiley \& Sons, Inc., New York, N.Y., 1982, pp. 163258.

7. Bažant, Z. P., and Asghari, A., "Computation of Age-Dependent Relaxation Spectra," Cement and Concrete Research, Vol. 4, pp. 567-579; Also see "Computation of Kelvin-Chain Retardation Spectra," Cement and Concrete Research, Vol. 4, 1974, pp. 797-806.

8. Bažant, Z. P., and Chern, J. C., "Double Power Logarithmic Law for Basic Creep of Concrete," Center for Concrete and Geomaterials, Northwestern University, Evanston, Ill., Dec., 1983 (also: submitted to Cement and Concrete Research)

9. Bažant, Z. P., Rossow, E. C., and Horrigmoe, G., "Finite Element Program for Creep Analysis of Concrete Structures," Proceedings of the 6th International Conference on Structural Mechanics in Reactor Technology (SMiRT6), Paris, France, Paper H2/1.

10. Bažant, Z. P., and Wu, S. T., "Dirichlet Series Creep Function for Aging Concrete," Journal of the Engineering Mechanics Division, ASCE, Vol. 99, No. EM2, Proc. Paper 9645, 1973.

11. Bažant, Z. P. and Wu, S. T. "Rate-Type Creep Law of Aging Concrete Based on Maxwell Chain," Materials and Structures, RILEM, 7, 1974, pp. 45-60.

12. Cost, T. M., "Approximate Laplace Transform Inversions in Viscoelastic Stress Analysis," American Institute of Aeronautics and Astronautics, Vol. 2, 1964, pp. 2157-2166.

13. Gamble, B. R., and Thomass, L. H., "The Creep of Concrete Subject to Varying Stress," Proceedings of the Australian Conference on the Mechanics of Structures and Materials, Adelaide, Australia, Paper No. 24, Aug., 1969.

14. Hanson, J. A., "A Ten-Year Study of Creep Properties of Concrete," Concrete Lab., Report No. SP-38, U.S. Dept. of the Interior, Bureau of Reclamation, Denver, Colo., July, 1953.

15. Harboe, E. M., et al., "A Comparison of the Instantaneous and the Sustained Modulus of Elasticity of Concrete," Concrete Lab. Report No, C-854, Div. of Engrg. Lab., U.S. Dept. of the Interior, Bureau of Reclamation, Denver, Colo. Engrg. Lab.,
Mar., 1958.

16. Hardy, G. M., and Riesz, M., "The General Theory of Dirichlet Series," Cambridge Tracts in Mathematics and Mathematical Physics, Cambridge University Press, $18,1915$.

17. Lanczos, C., "Applied Analysis," Prentice-Hall, Englewood Cliffs, N.J., 1964, pp. $272-280$.

18. L'Hermite, R. G., Mamillan, M., and Lefèvre, C., "Nouveaux résultats de recherches sur la déformation et la rupture du béton," Annales de l'Institut Techn. du Bâtiment et des Travaux Publics, Vol. 18, No. 207-208, 1965, pp. 323360 (see also International Conference on the Structure of Concrete, Cement and Concrete Association, London, England, 1968, pp. 423-433).

19. "Prediction of Creep, Shrinkage and Temperature Effects in Concrete Structures," Report No. ACI 209 R-82 by ACI Committee 209, Subcommittee II, chaired by D. J. Carreira, ACI Special Publication SP-76, "Designing for the Effects of Creep, Shrinkage and Temperature," Proceedings, A. Pauw Symposium held at ACI Convention, Houston, 1978, American Concrete Institute, Detroit, Mich., 1982, pp. 193-300.

20. Rostasy, F. S., Teichen, K. Th., and Engelke, H., "Beitrag zur Klärung der Zusammenhanges von Kriechen und Relaxation bei Normal-beton," Amtliche Forschungs-und Materialprüfungsanstalt für das Bauwesen, Otto-Graf-Institut, Universität Stuttgart, Strassenbau und Strassenverkehrstechnik, Heft 139, 1972. 21. Schapery, R. A., "Approximate Methods of Transform Inversion for Visco- elastic Stress Analysis," Proceedings of the 4th U.S. National Congress of Applied Mechanics, (held at Berkeley, Calif.), Vol. 2, American Society of Mechanical Engineers, 1962, pp. 1075-1085.

22. Taylor, R. L., Pister, K. S., and Goudreau, G. L., "Thermomechanical Analysis of Viscoelastic Solids," International Journal of Numerical Methods in Engineering, Vol. 2, 1970, pp. 45-60.

23. Williams, M. "The Structural Analysis of Viscoelastic Materials," American Institute of Aeronautics and Astronautics, Vol. 2, 1964, pp. 785-808.

24. Zienkiewicz, O. C., Watson, M., and King, I. P., "A Numerical Method of Viscoelastic Stress Analysis," International Journal of Mechanical Science, Vol. 10, 1968, pp. 807-827.

\section{APPENDIX II.-Notation}

The following symbols are used in this paper:

$a, q=$ constants for determining retardation time (Eq. 14);

$E_{\mu}=$ spring modulus of $\mu$ th Kelvin unit (Eqs. 10, 11, 13)

$J\left(t, t^{\prime}\right)=$ creep function (compliance) $=$ strain at time $t$ caused by unit uniaxial stream acting since age $t^{\prime}$ (Eqs. 1, 2);

$N=$ number of Kelvin chain units;

$t=$ time from casting of concrete, in days

$t^{\prime}=$ time of application of constant stress;

$y_{\mu}=$ functions representing reduced time

$\epsilon, \sigma=$ uniaxial strain and stress (Eq. 1);

$\epsilon^{0}=$ prescribed stress-independent strain representing shrinkage and thermal dilation (Eq. 1);

$\epsilon_{\mu}, \sigma_{\mu}=$ hidden strains and hidden stresses in Kelvin units (Eqs. 8 9, 10);

$\eta_{\mu}=$ viscosity of $\mu$ th dashpot in Kelvin units (Eqs. 10, 11, 13);

$\tau_{\mu}=$ retardation times $(\mathrm{Eq} .14)$; and

$\gamma_{\mu}=$ hidden strains in $\mu$ th spring in Kelvin unit (Eqs. 4, 7, 8).

\section{Subscripts}

$k, m=$ sampling points in $\log t^{\prime}$ and $\log t$ scale; and

$\mu=\mu$ th Kelvin unit. 